\title{
Snapshop-An Image Based Search Application
}

Karan D. Argade ${ }^{1}$, Dhanashree M. Gaware ${ }^{1}$, Prajkta S. Umap ${ }^{1}$, Savita P. Nalawade ${ }^{1}$, Snehal Baravkar ${ }^{2}$

${ }^{1}$ Department of Computer Engineering, Shree Ramchandra College of Engineering, Pune, Maharashtra, India

${ }^{2}$ Assistant Professor, Department of Computer Engineering, Shree Ramchandra College of Engineering, Pune, Maharashtra, India

\section{Article Info}

Volume 8, Issue 3

Page Number : 691-697

\section{Publication Issue}

May-June-2021

\section{Article History}

Accepted : 10 June 2021

Published : 17 June 2021

\section{ABSTRACT}

E-commerce has been growing rapidly over the past few years, Peoples uses them to buy and sell products. Since In these offline stores, they face many problems such as inability. For many online customers, image recognition of clothing and to identify the style, the color, and, in fact, it is a challenge to the sophistication of the fashion industry. In e-commerce, the online platform primarily offers text-based search capabilities. They can search many product searches, but they cannot manage searches based on product features, for example, colors or t-shirt patterns. Often, it is difficult for the user to make this determination features when searching for a product. Furthermore, an increasing number of consumers are depending on social media to make purchasing decisions. Consumers are trying to discover what is going on right now and are looking for the same things. This brings us to the need for a visual commerce platform, or a plan, which recommends products based on users, provided that the product images. The database uses a flexible neural network. You extract data using this deep neural network of image recognition, pattern matching and are very effective in testing fabric prediction.

Keywords : Image recognition, convolutional neural network, Pattern Recognition

\section{INTRODUCTION}

Clothing is an important part of your everyday life. Our clothes reflect us as individuals and as a society. In response to the growing demand for clothing increases, researchers are working to prepare the software to update the in our day to day life. Therefore, to clothes, to search for information in the application is useful in the modern world of fashion. There are large amounts of data, a good job, clothes, and world-class photos were taken in a variety of settings, including retail stores, the photos were taken on the street, and the carefully chosen outfits. Figure 1 shows some examples of the clothing, which is the largest on-line mode the library. Photo by. The true definition of a special or similar device related to the subject of the collection of the information from the large quantities is not a problem. Therefore, it is important to research is devoted to the classification of the clothes: to predict the quality and returns of 
the products on the basis of their increase in the prices of the sector. In order to do this, the user may need to provide the questions for the photos, the fabric, and the system will search for similar images, databases, and retrieving items such as clothes, in a photo poll.

Image Recognition is so helpful in an everyday fashion that online shoppers can take photos to search for anything, getting search results without typing is the main function of viewing a photo that acts as a search engine. The problem of defining the image of clothes can be classified as a separate question. The basic methods of knowing the image of the garments can be divided into different machine learning algorithms. Image recognition is used to perform various tasks such as tagging photo content with tags, conducting image content search, self-driving cars, directing robots, and avoiding the risk of big data.

Machine learning consists of a variety of models including pattern matching and recognition techniques. Pattern matching is the process of identifying or checking whether a token or letter or some sequence of data or a picture is present in the provided data. Comparison of educational patterns with visual patterns. Potting is an algorithm function that detects predefined patterns between raw data sequences or tokens or processed characters. Find new ways Abstract pattern matching techniques are similar for the two types of patterns and determine whether they are the same or not both are the same and both are different.

The image recognition system includes the standard operating functions outlined below.

Image Acquisition: The picture is taken with the help of a variety of units, with photos. A variety of sensors are available such as light sensors, ultrasonic, radar, and more. Depending on the sensor type, to reconstruct the image, which may be two- dimensional, three-dimensional, or type.Depending on the sensor type, to reconstruct the image, which may be two-dimensional, three-dimensional, or type.

- Pre-processing: Before you delete an object in the image processing, which are based on certain assumptions and conditions, as image format, image resolution, color, size, etc, Some of the initial experience with the features include a histogram of the equilibrium conversion, noise reduction, etc

- Feature Feature: This is one of the most important steps in image recognition, as all of the most important features in the image is derived from this category. A few of the most common file types related are:

- Complex features

- $\quad$ Eigenvectors

\section{- $\quad$ Local Interest Points}

- Segmentation: While processing the image, different image areas are selected and suitable points for further processing appear. For example, separating an image into multiple areas of an image where each region contains the same object.

- Advanced Processing: In This section, which deals with information that is included in the section is in the shape of a sphere corresponding to the image size, or to a specific element. The entrance of the claims are valid only if they comply with the application or an offer of a model. After that, they measured the various parameters related to the object's size and shape.Finally, all of items are classified into several categories based on their properties.

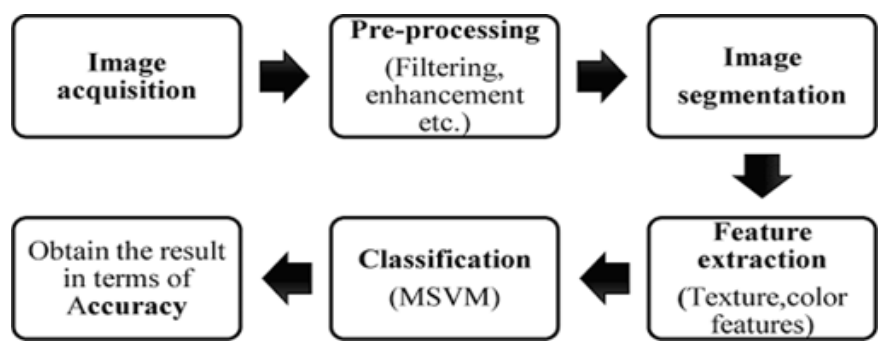

Figure 1. Image Recognition System 


\section{PROPOSED SYSTEM}

- Decision Making: This step is to make a final decision about whether there is a game or not within the application.

\section{RELATED WORK}

Over the years, Artificial Intelligence (AI) is replacing ordinary computers. It provided applications with many powerful capabilities in the form of machine learning computer algorithms and NLP which was previously almost impossible. With the advent of this technology, we have seen a lot of new things around us, especially in e-commerce as smart assistants.

In the 1960s, one of the core language understanding applications was ELIZA which was very effective in identifying phrases and displaying words from inputs, and providing pre-rendered responses to a meaningful discussion that solved specific issues Was doing, Jonathan Shih et al., (2010) Suggestions for the use of online descriptive text to test features and overall appearance. Y, c. Wahl et al., (2013) propose a pattern of using the learning trait to include better interpretation of the widely used clothing image in the computer viewing community.

Y.Bai et al. (2013) have proposed a network of deep neural activity for retrieval of text-based images. J. Tang et al. (2016) Proposed Multilayer Perceptron.

To jointly analyze the set of clothing images in the Semantic setting Wei Yang et al. (2015) proposed a mixed system of dress codes. It has developed a model for exploring the collection of images for wearing clothes simultaneously and annotation of smart pixels. Vissarut Surkarin et al. (2016) proposed a way to classify and identify clothing types using SURF in combination with LDP based on the BoF model.
The concept of machine learning is being used in variety of application domains such as predicting diseases, stock price prediction, weather forecasting and many more. It has minimized the computation efforts to a great extent. However, there is an area in machine learning which is rapidly growing i.e., Computer Vision. It is defined as the process of extracting, analyzing and understanding valuable information from an image or a sequence of images. Anecdotally, approximately $70 \%$ of the neural activity in the human brain is related to vision either directly or indirectly.

An important application of Computer Vision is Image Recognition. There is extensive research being done in this field of Image Recognition, based on a maxim that an image is worth a thousand words. However, on the world-wide web, converse may be true; it is easy to perform text based search on Google as compared to a visual search using an uploaded image which may return erratic results. With the easy availability of image data and a high rise in use of mobile devices with in-built cameras and internet connection, this field of image recognition in visual search will continue to grow in future.

The Conventional Neural Network (CNN) is an indepth learning algorithm capable of capturing image input, assigning values (readable tools and selections) to different elements/elements in the image, and separating them. In Connet, pre-processing is much smaller than other phase algorithms. While filters are made by hand with basic techniques, ConvNets can learn these filters/features with adequate training.

The structure of the Connet is similar to the structure of communication of neurons of the human brain and this organization is influenced by the visual cortex. In only a limited viewing field known as the receiving field, individual responses react to events covering the entire visual field, a collection of such fields 
passes. The simple CNN is depicted in fig. 2 shows the direct output.

The basic blocks of CNN are:

1. Convergence layer- A "filter" known as a "kernel" is transferred to an image looking at a few pixels at a time (eg $3 \times 3$ or $5 \times 5)$. The convolution function is a product of the dots defined by the weight in the pixel filter. Find summarized in the value of all pixel filter information.

2. Activation layer - Convectionary layer creates a much smaller size than the image. This feature distributes a non-linear launch layer to allow the network to train itself in the background. Sigmoid, SoftMax, Relu, and TANH have activating features.

3. Pool layer - "merge" means further reduction and reduction in the volume of the matrix. The filter is passed through the result of the previous layer and is called a number (usually range, this is peak pooling) from each value range. This helps the network learn faster, focusing on the most important details in each aspect of the image.

4. Fully integrated layer - standard format for multilayer perceptrons. Its inclusion is a velvety single representing the release of the previous layers. Working on a group of possibilities associated with something (eg dog, cat, bird)

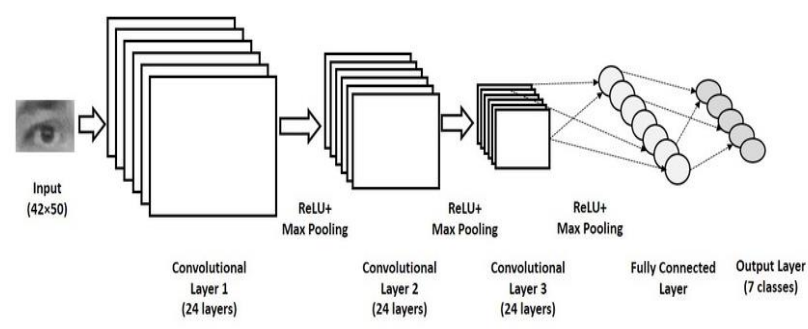

Figure 2. CNN's simple design for fashion MNIST

A common problem of computer viewing, especially in image processing, is that the image has certain features, features, or functions. Accordingly, several types of image recognition are available for various purposes:

- Object recognition: It is a way of viewing one or more previously studied objects or their classes if they exist in a uniform or three-dimensional space. For example, Google Goggles and Blippar are applications based on search engine optimization.

- Identification: It is used to identify a specific object. For example, pointing to someone's face, handwritten digital identification, etc.

Detection: It is used to view image data for a specific situation. For example, the discovery of untouchable tissue or cells in images in the field of medical science. It is sometimes used to obtain specific areas of interesting data from images to further analyze them using advanced computer techniques to achieve higher accuracy.

The state-of-the-art algorithm in image recognition relies on a system known as the Conversational Neural Network (CNN). ImageNet is used as a benchmark for acquisitions and divestitures, demonstrating the CNN offering. It is a visual acuity challenge that takes place extensively using millions of images from different classes. Several tests have proven that $\mathrm{CNN}$ in the ImageNet data set provides the most accurate results and performance that is closest to human response. However, there are some challenges that these algorithms fight for. These challenges relate to knowing something in the form of small ants in a flower stalk.

In addition, they have difficulty distinguishing images with filters similar to Instagram's filter filters. However, it is not at all difficult for people to see those pictures. However, viewing orbits with beautiful characters from a photograph can cause people a lot of trouble finding bird species, which can 
be easily identified using CNN. Some applications provided for image recognition are based on functions, such as:

- visual character recognition

- Image Recognition

- content search

- Post Balance

- 2D code reading

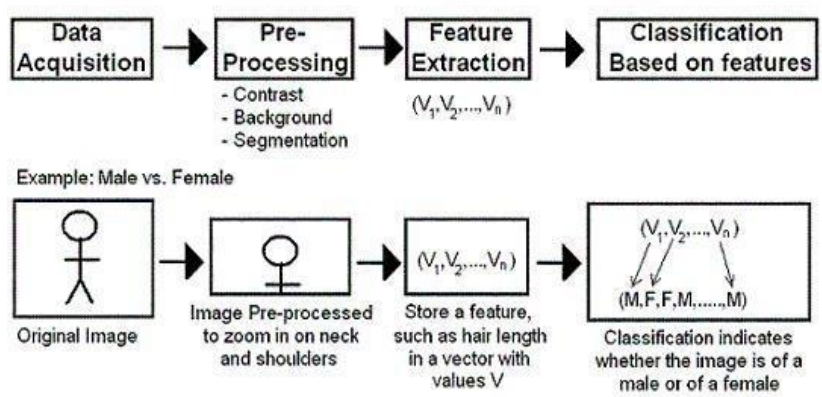

Figure 3. Proposed System Design

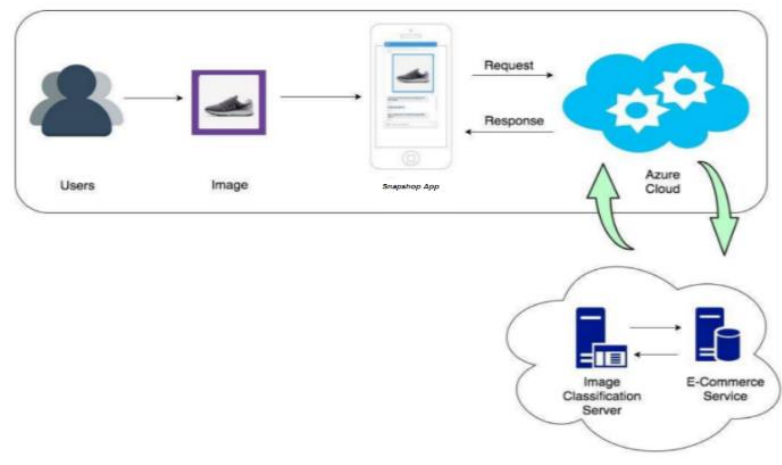

This Design consist of image recognition and machine learning algorithm which popularly known as CNN. This Design contains:

- Data Acquistion : It consist of Original Image of product.

This image consist of $\mathrm{t}$-shirt, etc.

- Pre-Processing : This stage contains various preprocessing techniques on the image like zoom-in or out, Contrast, Background of image,

Figure 2. Proposed System Architecture

This architecture is developed in the form of a pipeline due to the presence of multiple tasks such as conversational interface, image recognition and product retrieval, which are required to be performed in a sequential manner. Hence, each of these tasks is handled individually.

The overall architecture of this project consists of the following components:

- User Interface: It is a necessary part of any user based application. It helps in engaging users and providing them with a great experience.

- Middleware: This layer comprises of an Image Recognition based web service. Our whole project somewhat revolves around this service, which makes it one of the main components in our project.

Dataset: This layer cintains dataset which is provided by retailer.

\section{CONCLUSION}

In this study, we have tried to find the answer to our research question of building a visual shopping system using state-of-the-art visual image technology to improve the online shopping experience for users. We have suggested a new way of providing users with a relevant product list based on visual demand. The proposed application has two main features, namely, image recognition service and product retrieval service. Each of these components is an integral part of this app. However, the image recognition service is the basic functionality of the app that enables users to extract visual information from images that are 
difficult to define in words, for example, categories, product names, product color features.

With the widespread use of social networking sites like Facebook, Twitter, people are becoming more and more curious about the latest developments around them. They follow a wide variety of people, for example, their favorite celebrities. His purchasing decisions are heavily influenced by social media. Therefore, having a product search application is very powerful for finding the right products by just giving an image. Now, users have a better way to find their product manager. They no longer have to go to traditional texts based on texts. Instead, they can let the application find the right product for them. They only have to provide an image of the product they want.

\section{FUTURE SCOPE}

Based on our research, we have developed a fullyfunctional search engine, drive that delivers positive results. However, due to the limited scope of the project has not been able to work on it, for maximum accuracy and performance. Therefore, as part of a future project, we would like to develop, refine, and add other features. Some of them are described below:

- Training data training: We have seen how important the training data set is in building an accurate image classification model. During our experiments with pre-trained separators, we observed specific side effects that could be used as part of the solution. Therefore, it is very important to have a specific database with welldefined features. In addition, the database should contain data for most products, not just shoes to create a visual recognition service that can differentiate the various features found in ecommerce. To create this type of data, we will create a data conflict over multiple data sets to obtain a unified data source that includes multiple products

- Social Media Integration: In order to attract users to this program, we will post it on various social media sites such as Facebook Messenger, Slack, Skype, etc.

- E-commerce assets: With offline e-commerce, inventory in order to make the items related to the positions, which limits the ability to consume the purchase. For example, if a user wants to purchase a particular gene, they will have to be limited to the currently available options in the list.

- Multi-disciplinary awareness: It is unavoidable to have the ability to see many things based on the problem we are trying to solve. A user can't use an image that shows only one thing.

\section{REFERENCES}

[1]. G. Eason, B. Noble, and I.N. Sneddon, "On certain integrals of Lipschitz-Hankel type involving products of Bessel functions,"Phil.Trans. Roy. Soc. London, vol. A247, pp. 529-551, April 1955. (references)

[2]. J. Clerk Maxwell, A Treatise on Electricity and Magnetism, 3rd ed., vol. 2. Oxford: Clarendon, 1892, pp.68-73.

[3]. I.S. Jacobs and C.P. Bean, "Fine particles, thin films and exchange anisotropy," in Magnetism, vol. III, G.T. Rado and H. Suhl, Eds. New York: Academic, 1963, pp. 271-350.

[4]. K. Elissa, "Title of the article, where it is known as," highly-cited publications.

[5]. R. Nicol, "the title of the article, with a capital letter only for the first word', J. Bear the name. The abbreviation of the press at the time.

[6]. Y. Yorozu, M. Hirano, K. Oka, Y. Tagawa, "Electron spectroscopy studies on magnetooptical media and plastic substrate interface, IEEE Transl. J. Magn. Japan, vol. 2. 740-741, 
August 1987 [Digests 9th Annual Conf. Cite this article as :

Magnetics Japan, p. 301, 1982].

[7]. M. Young, The Technical Writer's Handbook. Mill Valley, CA: University Science, 1989

Karan D. Argade, Dhanashree M. Gaware, Prajkta S. Umap, Savita P. Nalawade, Snehal Baravkar,

[8]. Peharz, R., Vergari, A. Stelzner, K., Molina, A., Kersting, K, \& Ghahramani, Z. (2018). Probabilistic deep learning with the help of the random, the sum of the network. Preprint Archive:1806.01910. "Snapshop-An Image Based Search Application", International Journal of Scientific Research in Science and Technology (IJSRST), Online ISSN : 2395-602X, Print ISSN : 2395-6011, Volume 8 Issue 3, pp. 691-697, May-June 2021. Available at

[9]. Q. Dong, S. Gong, and X. Zhu ' Multi-task with the curriculum of the deep learning network attributes, " in Proc. IEEE Winter Conf. Appl. Comp. Kind of. (WACV), march. In 2017, the s. 520_529.

[10]. R. B. Girshik, J., Donahue, T., Durrell, J. P., "Adaptive convolutional networks for accurate object detection and segmentation", IEEE Trans. Model, Anal. Max. Intellige., no. 38, No. 1, pp. 142_158, Jan. 2016.

[11]. R. Li, F. Feng, I. Ahmad, and X. Wang, "Retrieving real world clothing images via multi-weight deep convolutional neural networks," in Cluster Computing. New York, NY, USA: Springer, Jul. 2017.

[12]. S. Chen, H. Wang, F. Xu, and Y.-Q. Jin, "Target classification using the deep convolutional networks for SAR images," IEEE Transactions on Geoscience and Remote Sensing, vol. 54, no. 8, pp. 4806-4817, 2016

[13]. Seo Y, \& Shin, K. S. (2019). A hierarchical convolutional neural network to classify fashion images. Expert systems, applications, 116, 328-339.

[14]. Xiao, H., Rasul, K., \& Vollgraf, R. FashionMNIST: a Novel Image Dataset for Benchmarking Machine Learning Algorithms. Aug. 28, 2017. Arxiv preprint cs.LG/1708.07747.

[15]. Z. Huang, Y., Yu, J., Dong, H. Liu, ' 'Effective, way of the recognition is based on a extreme machine learning", IEEE Trans. Cybern., no. 47, No. 4, pp.920_933, Nov. 2017.

doi : https://doi.org/10.32628/IJSRST2183151

Journal URL : https://ijsrst.com/IJSRST2183151 